\title{
Embryology of the Biliary Tract
}

\author{
Hisami Ando \\ Department of Pediatric Surgery, Nagoya University Graduate School of Medicine, Nagoya, Japan
}

\section{Key Words}

Bile duct development · Biliary tract • Embryology •

Extrahepatic biliary ducts $\cdot$ Hepatopancreatic ducts

\begin{abstract}
A hepatic diverticulum appears in the ventral wall of the primitive midgut early in the 4th week of intrauterine life in the development of the human embryo. This small diverticulum is the anlage for the development of the liver, extrahepatic biliary ducts, gallbladder, and ventral pancreas. By the 5th week, all elements of the biliary tree are recognizable. Marked elongation of the common duct occurs with plugging of the lumen by epithelial cells. Recanalization of the lumen of the common duct starts at the end of the 5th week and moves slowly distally. By the 6th week, the common duct and ventral pancreatic bud rotate $180^{\circ}$ clockwise around the duodenum. Early in the 7th week, the bile and pancreatic ducts end in closed cavities of the duodenum. Between the early 8th and 12th week, hepatopancreatic ducts have both superior and inferior orifices. Of these two orifices, the inferior one is usually suppressed. The muscle of the sphincter of Oddi develops from a concentric ring of mesenchyme surrounding the preampullary portion of the bile and pancreatic ducts. At about the 10th week, the muscle of the sphincter of Oddi undergoes differentiation. In the 16 th week, the muscularis propria extends from just outside the fenestra to the upper end of the ampulla. By the 28th week, the musculus proprius is differentiated almost to the distal end of the ampulla.

Copyright $\odot 2010$ S. Karger AG, Basel
\end{abstract}

\section{Development of the Bile Duct}

A hepatic diverticulum appears in the ventral wall of the primitive midgut early in the 4 th week of intrauterine life in the development of the human embryo [1]. This small diverticulum is the anlage for the development of the liver, extrahepatic biliary ducts, gallbladder, and ventral pancreas. In the 4 th week, two buds can be recognized in the hepatic diverticulum $[2,3]$. The cranial bud becomes the liver and the extrahepatic biliary tree. The caudal bud develops into superior and inferior buds. From the superior bud, the gallbladder and cystic duct appear, and the right and left ventral pancreas develops from the inferior bud. By the 5th week, all elements of the biliary tree are recognizable [2, 4] (fig. 1). Meanwhile, bile canaliculi differentiate from hepatic cells. The terminal bile ducts grow out into the mesenchymal tissue of the septum transversum, which will produce the fibrous architecture of the liver, and the development of an intrahepatic duct system is completed by the 10th week [4-6]. Marked elongation of the common duct occurs with plugging of the lumen by epithelial cells. Recanalization of the lumen of the common duct starts at the end of the 5 th week and moves slowly distally [7]. The lumen of the common duct extends into the cystic duct by the 7 th week, but the gallbladder remains solid until the 12th week. By the 6 th week, the common duct and ventral pancreatic bud rotate $180^{\circ}$ clockwise around the duodenum. After completion of the rotation, the entrance of the common bile duct into the left posterior surface of the duodenum can be seen. Early in the 7th week, the bile and pancreatic ducts end in closed cavities of the duodenum

Hisami Ando, MD, PhD

Department of Pediatric Surgery, Nagoya University Graduate School of Medicine 65 Tsurumai-cho, Showa-ku

Nagoya 466-8560 (Japan)

Tel. +81 52744 2958, Fax +81 52744 2980, E-Mail hando@med.nagoya-u.ac.jp 


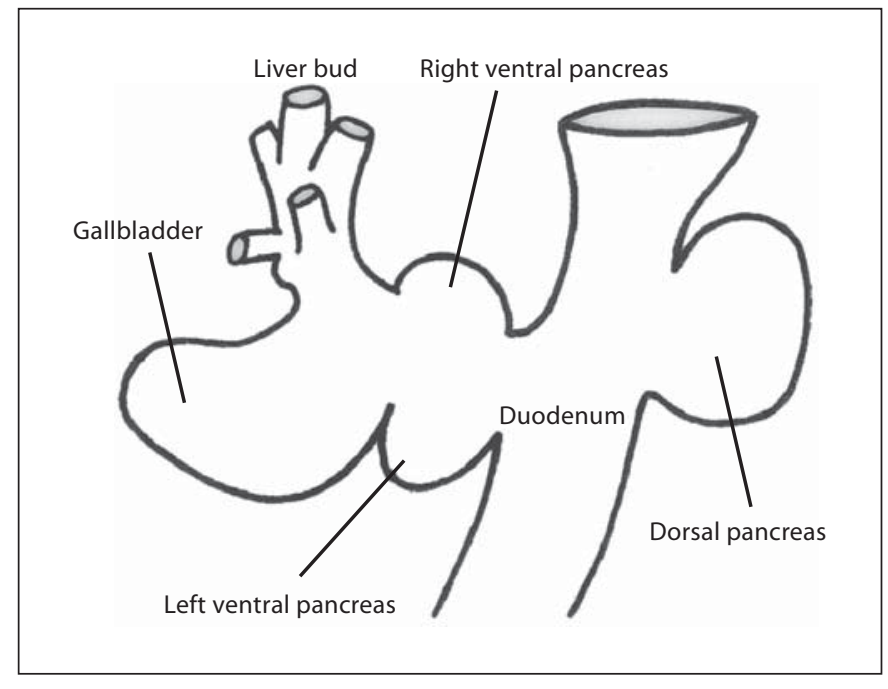

Fig. 1. Development of the hepatic diverticulum (5th week). The diverticulum is the anlage for the development of the liver, extrahepatic biliary ducts, gallbladder, and ventral pancreas.

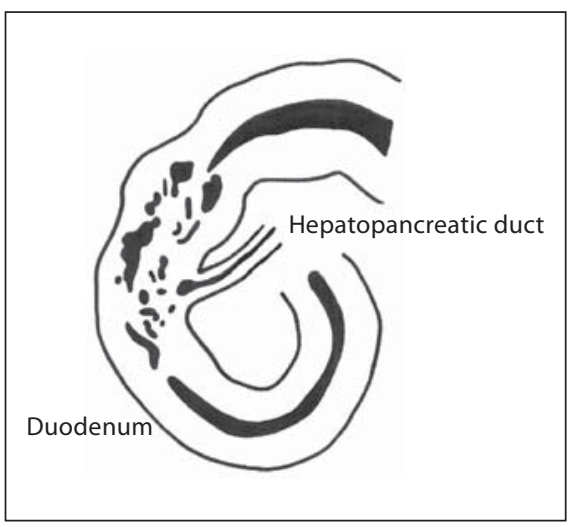

Fig. 2. Graphic reconstruction of the duodenum and hepatopancreatic duct of the 6th week (modified from Boyden et al. [8]). The bile and pancreatic ducts end in closed cavities of the duodenum.

(fig. 2). However, the cavity between the orifices of the dorsal pancreas and common bile duct is completely obliterated for the active epithelial proliferation of the developing common bile duct. Between the end of the 7th and beginning of the 8th week, the bile duct develops two channels, and the ventral one continues into the lower segment $[3,7,8]$. Still in the 8 th week, in the distal segment of the duodenum, two parallel channels and vacuoles are still present [6].

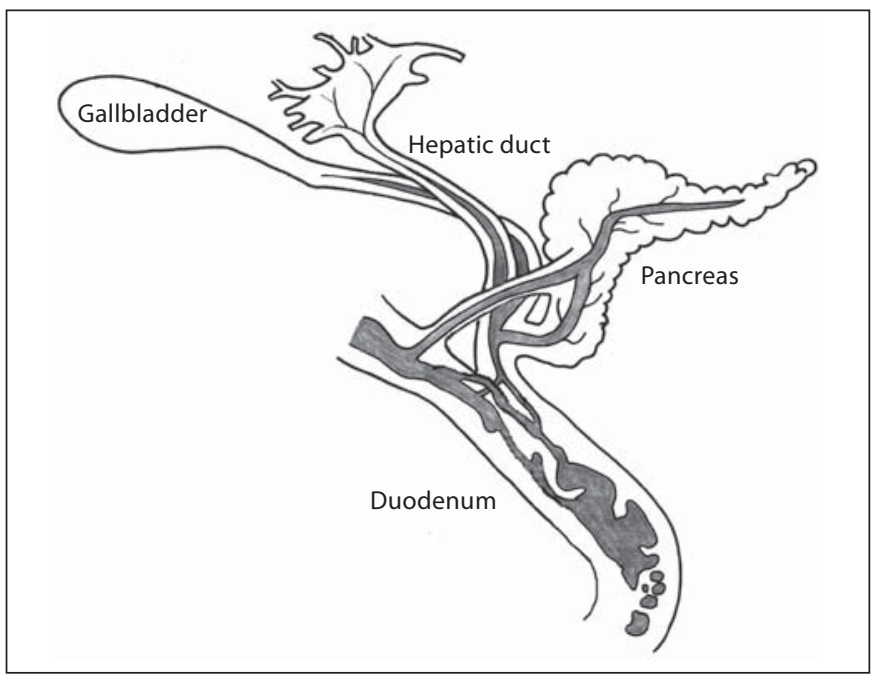

Fig. 3. Graphic reconstruction of the epithelial anlage of the duodenum and hepatopancreatic duct of the 8 th week. Two channels appear in the developing biliary system, each of which joins up separately with the two duodenal channels.

\section{Development of the Duodenum}

In the 4 th week, the epithelium of the duodenum undergoes active proliferation, which completely occludes its lumen $[2,3]$. At the beginning of the 6 th week, the primitive duodenum contains a small lumen. From the end of the 7 th week to the 8 th week, the duodenum increases in diameter and the vacuoles coalesce and begin to restore the patency of the lumen by epithelial proliferation and vacuolation $[7,8]$. The coalescence of vacuoles initially produces two parallel channels in the duodenum while, at the same time, two channels appear in the developing biliary system, each of which joins up separately with the two duodenal channels $[6,8]$. From the early 8 th week to the 12th week, hepatopancreatic ducts have both superior and inferior orifices. A superior orifice opens into the dextral cavities of the gut and an inferior orifice opens into the sinistral cavity [3, 7] (fig. 3). Of these two orifices, the inferior one is usually suppressed, but it may persist in the form of a blind pocket or a patent orifice.

\section{Development of the Sphincter of Oddi}

By the 7th week, further elongation of the hepatopancreatic duct pushes the junction of bile and pancreatic ducts out to the level at which the intestinal muscle is forming. In the 8th week, the site of the junction is ini- 
tially retracted through the slit of the duodenal wall, and soon comes to lie within the submucosa of the intestine [7]. The muscle of the sphincter of Oddi develops from a concentric ring of mesenchyme surrounding the preampullary portion of the bile and pancreatic ducts [9]. At about the 10th week, 4 weeks after the intestinal muscle has formed, the muscle of the sphincter of Oddi undergoes differentiation. The sphincter choledochus inferior appears first around the bile duct, which is carried away from the intestinal muscle, setting it up as an independent agent. After that, the sphincter choledochus supe- rior is formed. By the 12th week, the bile and pancreatic ducts enter the duodenum obliquely, as a result of which the fibers appearing on the mucosal side of the bile duct seem to extend between the two margins of the transverse slit in the circular muscle layer of the duodenum. At this time, the liver begins to secrete bile that flows via the extrahepatic biliary tree into the duodenum [9]. In the 16th week, the muscularis propria extends from just outside the fenestra to the upper end of the ampulla. By the 28th week, the musculus proprius is differentiated almost to the distal end of the ampulla.

\section{References}

1 Bremer JL: Description of a $4 \mathrm{~mm}$ human embryo. Am J Anat 1906;5:459-480.

$>2$ Kanagasuntheram R: Some observations on the development of the human duodenum. J Anat 1960;94:231-240.

-3 Jona JZ, Belin RP: Duodenal anomalies and the ampulla of Vater. Surg Gynecol Obstet 1976;143:565-569.

4 Lindner HH, Green RB: Embryology and surgical anatomy of the extrahepatic biliary tract. Surg Clin North Am 1964;44:12731285 .
Reid IS: Biliary-tract abnormalities associated with duodenal atresia. Arch Dis Child 1973;48:952-957.

6 Irving IM, Rickham PP: Duodenal atresia and stenosis: annular pancreas; in Rickham PP, Lister J, Irving IM (eds): Neonatal Surgery, ed 2. London, Butterworths, 1978, pp 355-370.

7 Schwegler RA, Boyden EA: The development of the pars intestinalis of the common bile duct in the human fetus, with special reference to the origin of the ampulla of Vater and the sphincter of Oddi. I. The involution of the ampulla. Anat Rec 1936-1937;67:441467.
8 Boyden EA, Cope JG, Bill AH: Anatomy and embryology of congenital intrinsic obstruction of the duodenum. Am J Surg 1967;114: 190-202.

-9 Schwegler RA, Boyden EA: The development of the pars intestinalis of the common bile duct in the human fetus, with special reference to the origin of the ampulla of Vater and the sphincter of Oddi. II. The early development of the musculus proprius. Anat Rec 1938;68:17-41. 